\title{
Backward and forward masking associated with saccadic eye movement
}

\author{
BARBARA A. BROOKS, DIANNE M. K. IMPELMAN, and JANET T. LUM \\ Department of Physiology, University of Texas Health Science Center, San Antonio, Texas 78284
}

\begin{abstract}
Visual masking effects on test flash thresholds were measured under real and simulated eye movement conditions to determine whether visual masking is primarily responsible for elevations in threshold that are sometimes associated with saccadic eye movements. Brief luminous flashes presented to the central retina before, during, and after saccades were masked by stimuli presented either pre- or postsaccadically. The amount and time course of masking were quantitatively dependent on stimulus parameters of intensity and temporal separation and were unaffected by eye movement parameters (amplitude, velocity, direction) as long as retinal stimulus conditions were constant. The duration of forward masking was longer than that of backward masking. When retinal conditions during saccades were mimicked while the eyes were held steady, masking interactions were identical to those obtained during real saccades. These results indicate that masking effects during saccades in ordinary environments are determined solely by the stimulus situation at the retina. Putative nonvisual, centrally originating saccadic suppression suggested by other authors is evidently not additive with visually determined masking during saccades.
\end{abstract}

The term "masking" refers to the competitive interaction between two or more visual stimuli presented close together in time and space. A stimulus is said to be masked when its visibility is diminished or eliminated by another stimulus. Masking has been extensively studied psychophysically, and there is a variety of interactions, depending upon the nature of the stimuli involved (e.g., see reviews by Kahneman, 1968; Weisstein, 1972). Several investigators have suggested masking as a primary determinant of the reduction in visual sensitivity that can be measured during voluntary saccadic eye movement (see reviews of "saccadic suppression" by Breitmeyer \& Ganz, 1976; Matin, 1974; Volkmann, 1976).

Since the fovea is aimed at successive objects of interest in ordinary environments, three temporal aspects of stimulation result in opportunities for masking interactions: (1) A clear, relatively stationary scene affects the retina during the fixation prior to a saccade, (2) blurred images briefly traverse the retina at saccadic velocities while the eye is in motion, and (3) a new set of sharp images accompanies the subsequent fixation. These transitions may occur two or three times a second during active viewing and are potential competition for each other. Among them, the images of both prior and subsequent fixation have been demonstrated to eliminate perception of the blur occurring during saccades; when the fixation images are avoided by illuminating the environment

This research was supported by NIH Grant RR07187 and NIH (NEI) Grant EY01497 to B. A. Brooks. D. M. K. Impelman was supported by NIH Postdoctoral Fellowship 5F 32EY05 191-03. Some of the results were presented to the Association for Research in Vision and Ophthalmology, Sarasota, Florida, May 1978. only during saccadic movement, the blurred world can readily be seen (Campbell \& Wurtz, 1978; related observations in the fixating eye by Corfield, Frosdick, \& Campbell, 1978). The importance of masking during ordinary viewing was also recently emphasized by Brooks, Yates, and Coleman (1980), who showed that vision for moving images during saccades may be as sensitive and accurate as during fixation if preand postsaccadic masking influences are carefully eliminated.

In this paper, we compare masking effects associated with real saccades with simulated eye movement conditions in which the retinal stimulation during saccades is mimicked while the eye is held steady. Significant differences between masking effects in these two almost identical stimulus situations would suggest that visual masking alone cannot explain saccadic suppression; similar effects would suggest that it can. We also explore some of the stimulus parameters that affect target-mask interactions when a mask is presented just before (presaccadic) or just after (postsaccadic) eye movement. Since the few existing studies of masking during eye movements stress highly structured stimuli or spatial interactions among stimuli affecting different areas of the retina (i.e., "metacontrast" in Davidson, Fox, \& Dick, 1973; Matin, Clymer, \& Matin, 1972; Wolf, Hauske, \& Lupp, 1978), our mask and target both are simple luminous stimuli that affect the same retinal location. Particular attention is paid to the time course of masking in relation to the eye movement.

The results indicate that there are no significant differences regarding masking interactions between the steady (or fixating) eye and the eye executing a 
voluntary saccade when the stimulus situation at the retina is the same in both conditions. Furthermore, masking interactions were quantitatively predictable based on the literature regarding masking in the fixating eye. ${ }^{1}$

In addition, the masking interactions we measured appeared unaffected by the introduction of highfrequency gratings in the mask, suggesting that our stimuli were preferentially affecting a single set of visual "channels" (the low spatial frequency or " $Y$ " channels of the literature). This implies some functional independence during ordinary eye movement between visual channels selective for high and low spatial frequencies.

\section{METHOD}

Mask and target stimuli were back-projected onto a $116 \times 100 \mathrm{deg}$ (visual angle) gray lenticular lens screen that was ambiently illuminated at $-2.0 \log \mathrm{fL}$. The projection system for the masking stimuli was located approximately $9 \mathrm{ft}$ behind the lens screen; the target projector was moved to change the stimulus position but remained about $3 \mathrm{ft}$ from the screen. Both systems were at the eye level of the observer.

The target was a 2.2-deg circular spot slightly defocused by approximately .3 D; it was flashed for $10 \mu \mathrm{sec}$ by a Grass PST-2100 strobe lamp mounted behind the optics of a GAF slide projector. Each test flash presentation was triggered by a Grass PS22 photostimulator and was monitored by a phototransistor placed in its light path; the phototransistor pulse was displayed with the EOG signal on a Tektronix D13 dual beam storage oscilloscope. Target characteristics were selected for minimal spatial frequency components, while allowing adequate spatial and temporal separation from the mask during 20 -deg saccades of 50 -msec duration (see Figure 1).

The mask was a $4 \times 4$ deg luminous patch defocused by approximately . $3 \mathrm{D}$ and projected via a mirror galvanometer system (General Scanning CCX100) that controlled its position as well as the direction and velocity of its movement. Neutral density filters were used to set the luminance level of mask and target stimuli. Luminance measurements were made at the beginning of each experimental session with a Tektronix narrow angle 1 deg luminance probe (Model J6523) placed at the position of the subject's cornea.

Figure 1 diagrams the method used to study masking caused by an image fixated before or after saccadic movement. The mask affected the center of gaze for $1 \mathrm{sec}$ during the fixation period either before or after eye movement. In the lower panel (presaccadic mask), the onset of movement results in the removal of the mask from the center of gaze, providing a potential forward mask for targets presented at the same retinal position during and after the saccade. Fixation of the mask at the end of the saccade (postsaccadic mask in upper panel) provides a potential backward mask for targets presented before and during the saccade. The position of the target in the diagram depicts a target flash occurring approximately midway through the saccade and affecting the center of gaze.

Saccadic eye movements were recorded by Beckman $\mathrm{Ag} / \mathrm{AgCl}$ skin electrodes placed on the outer canthus of each eye and differentially amplified by a Tektronix amplifier with 3-dB attenuation at 1 and $300 \mathrm{~Hz}$. The EOG voltage triggered the horizontal sweep of a storage oscilloscope. An output pulse gated from the oscilloscope's horizontal sweep generator simultaneously triggered a Grass S88 stimulator whose output to the photostimulator triggered the test flash presentation. The delay setting on the stimulator was used to present the test flash at selected times during and after the eye movement. The target position during eye move-

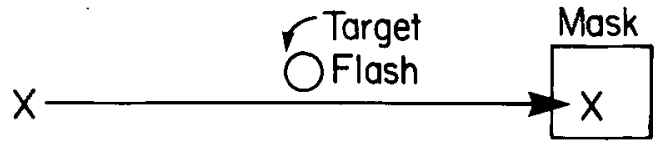

Postsaccadic Mask

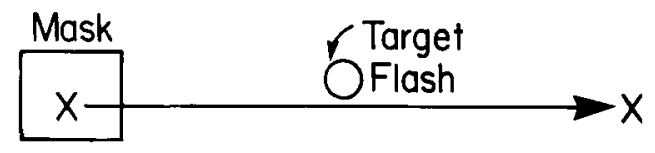

\section{Presaccadic Mask}

Figure 1. Schema of stimulus conditions for pre- and postsaccadic masking. Flxation areas are denoted by xs, and the arrows represent eye movements from left to right. The rectangle represents a $4 \times 4$ deg masking patch projected onto anlform background of $-2.0 \log$ fL. The target is depicted by the small circles. Postsaccadic mask (top): The target is fashed through a 20-deg 50-msec saceade, and its image falls on the central retian. About 25 msec Iater, the mask affects the same retinal location at the termination of the saccade. If the target is above threshold, it is percelved against the mask. The eye fixates the mask for approximately 1,000 msec. Presaccadic mask (bottom): On command to fixate, the observer steadily regards the mask at left. The saccade is made away from the mask, and the target is fashed as the center of gaze passes over Its position, about 25 msec later. If the target is above threshold, it is perceived at the fixation area at the end of the eye movement.

ments was adjusted so that it always occurred at the central retina, regardless of its temporal relation to the eye movement. Dim, vertical fixation lines located 20 deg above the center of gaze were occasionally used to guide eye movements. The lines had no effect on the data.

In order to present the target before the eye movement, an auditory cue to start the saccade was given and the target was triggered with a preset delay within the saccadic reaction time. The subject's reaction times (ranging from about 125 to $250 \mathrm{msec}$ ) were not always predictable, and therefore the timing of the target varied from trial to trial. Target timing and subject responses were recorded in 10-msec time bins. These intervals were determined by monitoring the time at which each phototransistor (flash) pulse occurred before the onset of the EOG on the oscilloscope screen. Thresholds for different times before the eye movements were defined as that luminance that was seen on $50 \%$ of its presentations during a given interval.

Stimulation at the retina during saccades was simulated by moving the mask across the visual field while the eyes were held steady. The mask movement was produced by applying a voltage ramp to the mirror galvanometer system; the rise time and amplitude of the ramp were selected to match the duration and amplitude of the saccadic eye movement as closely as possible. The memory oscilloscope was triggered from the voltage ramp to produce the target's appearance at the central retina at appropriate times relative to mask the movement. The target could be activated before the mask movement with various preset delays by the Grass stimulator. The steady eye position was monitored by observing the EOG on the monitor scope (accuracy within approximately 1 deg of visual angle).

\section{Procedure}

Observers included three adult females (D.I., B.B., and J.L.) and one adult male (J.M.). B.B. wore corrective lenses; D.I., 
J.M., and J.L. required none. All observers had had previous experience with psychophysics; one (J.M.) was naive regarding the experimental purposes.

Observers were seated facing the lens screen at a table that supported a chinrest. Fifteen minutes of dark adaptation preceded each experiment. Single trials were initiated by a "ready" signal from the experimenter, followed by a "fixate, go" command. "Fixate" indicated that the observer should steadily regard a designated area of the screen. The "go" command followed the "fixate" command by approximately $2-3 \mathrm{sec}$ and signified to saccade from one fixation area to another or signaled the onset of mask movement while the eye continued to be held steady (simulated saccadic condition). Immediately after the saccade or mask movement, the observer reported whether or not the target had been detected (yes/no).

Both the target-mask interval (T-MI) and target intensity were varied in experiments in which the time course of threshold change was measured. In some experiments, the T-MI was set and target intensity was varied until a threshold ( $50 \%$ detection) was found. In others, the mask and target luminance were set and the T-MI was varied until the target was just visible ( $50 \%$ detection).

An experimental run involved a series of 25 or more target presentations at a single intensity and preset interval, randomly interspersed with 20-30 control trials on which no target was presented. Observers were trained to perform at $90 \%$ correct or above on control trials, thus maintaining a consistent and stringent subjective response criterion. This procedure has been shown to yield results equivalent to the double-alternation forced-choice method when using experienced observers (see Appendix). After each run, the observer rested; about five runs could be achieved in a single sitting. Target intensities and target-mask intervals were unknown to the observer and were randomly changed for each run. Frequently, the intensity of T-MI yielding $50 \%$ detection could be quickly bracketed in preliminary trials, so that threshold could be determined in one or two runs.

Prior to each saccadic experiment, the position of an easily visible flashed target was adjusted so that the observer reported seeing it at the center of gaze. The target was first positioned at an angular distance from the initial fixation position, given by the product of the eye movement velocity and its timing after eye movement onset. If this calculation did not result in a report of "I saw it at the center of gaze" (i.e., on the fovea), further slight positional adjustments were made. In our conditions, the subject perceived the target at the postsaccadic fixation area, and not in its actual position on the lens screen. This is probably due to the latency of neural elements affected by the target (see also Brooks, Impelman, \& Lum, 1980). It requires $20-40 \mathrm{msec}$ for retinal processing alone and more time before the neural correlate of the stimulus reaches the cortex. Therefore, it was probably most usually the case that the saccade had ended before conscious perception of the target had occurred, with the consequence that the target was perceived against the stationary postsaccadic background.

\section{RESULTS}

\section{Target Thresholds in the Absence of Masks}

A background luminance of $-2.0 \log \mathrm{fL}$ was used throughout this study because thresholds for the target presented during fixation and saccades in the absence of any intentional masking stimulus were similar. A lack of significant difference between thresholds associated with eye movements and fixation on dim scotopic backgrounds had also been reported by Brooks and Fuchs (1975), Brooks, Impelman, and Lum (1980), Mitrani (1971), and Richards (1969). These results are in disagreement with the results of one other study (Riggs, Merton, \& Morton, 1974) and the interpretation of several other authors (e.g., Volkman, Riggs, Moore, \& White, 1978), who have suggested that differences in psychophysical method might be responsible for the disagreement. We therefore repeated our observations, using a doublealternation forced-choice method as well as the modified yes-no method described earlier in the procedure. No differences between saccadic and fixation data were found by either method for our experienced observer (B.B.), while a slight but not significant (.08 log unit) elevation in threshold was found during saccades for the less experienced observer (J.L.), while using the double-alternation forced-choice method. These results are detailed in the appendix to this paper and in no way alter the data discussed below.

\section{Masking of the Target}

Changes in sensitivity for the target flash caused by a presaccadic or postsaccadic masking stimulus are seen in Figures 2 and 3 for Subject B.B. A pre-

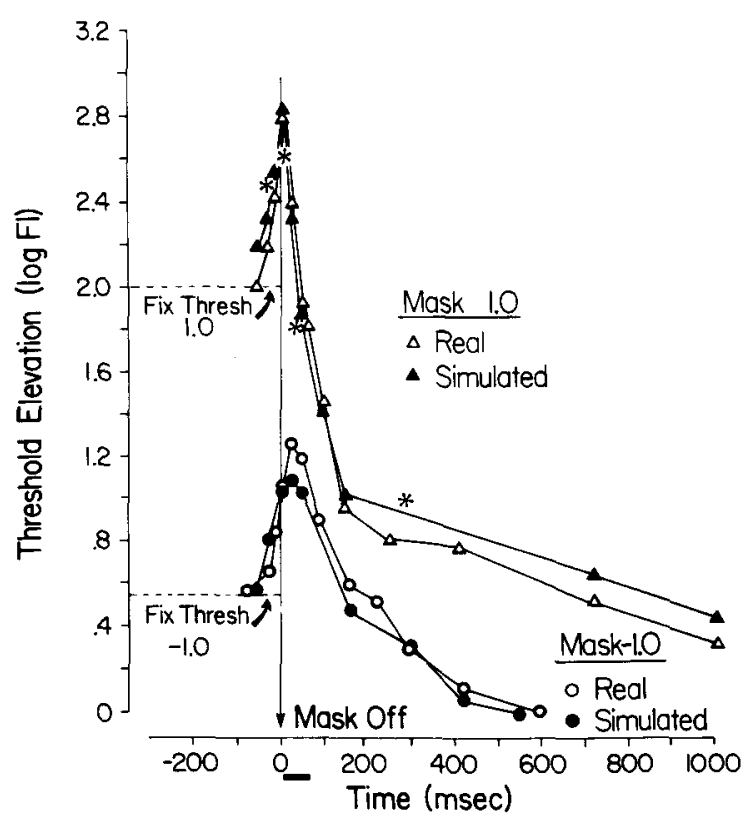

Figure 2. Changes in target threshold accompanying a presacendic mask. Ordinate: Elevation of target threshold relative to its value on the -2.0 -log-fL background without the mask (shown as zero). Abscissa: Time (msec) relative to the onset (depicted as 0 ) of eye or mask movement. The onset of movement results in a stepwise Iuminance decrease at the center of gaze ("mask off," downward pointing arrow). Duration of the 20 -deg eye (or mirror) movement resulting in the retinal trajectory of the mask is shown by the black line. All data were obtained from Subject B.B. Real saccade data are shown as open symbols; simulated saccade data obtained during eye steady are shown as filled symbols. Mask intensity for upper curves (triangles) $=1.0 \mathrm{log} \mathrm{fL}$, for lower curves (circles) $=-1.0 \mathrm{log}$ fL. Thresholds during steady regard of masks are indicated by the dotted horizontal lines (Fix Thresh). Asterisks show data obtained when the mask was a vertical grating at 14 cycles/deg, with a space-averaged luminance of $1.0 \mathrm{log} \mathrm{fL}$. Performance on control trials (no target) was $\mathbf{9 0 \%}$ or better (not shown). 


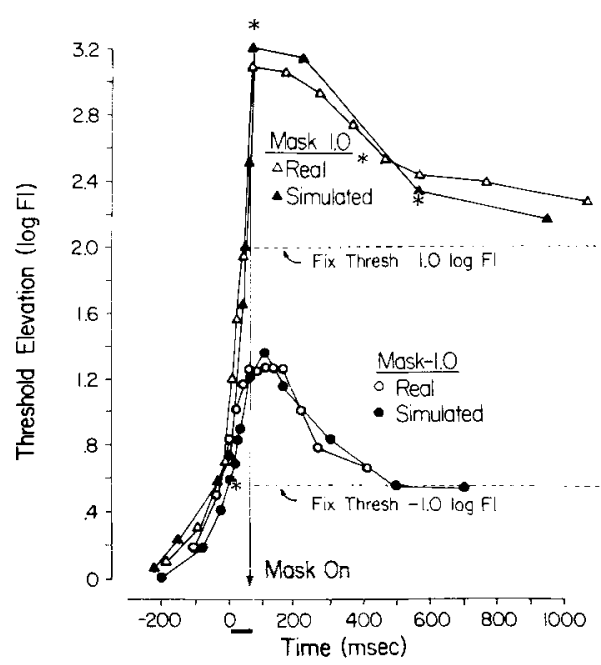

Figure 3. Changes in target threshold accompanying a postsaccadic mask. Ordinate: Conventions as in the last figure. Abscissa: Conventions as in the last figure, except that the mask affects the center gaze at the termination of movement ("mask on," downward pointing arrow). The duration of the mask's retinal trajectory is shown by the black line. All data were obtained from Subject B.B. Asterisk shows data obtained when the mask was a grating at 14 cycles/deg, with a space average luminance of $1.0 \mathrm{log}$ fL. Performance on control trials (no target) was $90 \%$ or better.

saccadic mask is equivalent to a step decrease in luminance at the center of gaze at the onset of eye or mask movement (Figure 2), while a postsaccadic mask causes an incremental step change at the termination of movement (Figure 3). Threshold elevations are plotted relative to the value measured during steady gaze at the background without the mask $(0$ on the ordinate). Each data point is the target luminance that yielded $50 \%$ frequency of seeing at the T-MI plotted on the abscissa. Zero msec represents the onset of eye or mask movement. Arrows show the time of "mask off" (Figure 2) or "mask on" (Figure 3) at the center of gaze.

The test situation of Figure 2 corresponds to the lower panel of Figure 1. Results are shown for two mask intensities, $-1.0 \log \mathrm{fL}$ and $1.0 \mathrm{log} \mathrm{fL}$, which were 1.0 and $3 \log$ units, respectively, above background luminance. The horizontal dotted lines indicate thresholds during steady gaze at the center of the mask ("Fix Thresh" 1.0 and -1.0 ). Real and simulated saccadic data are represented by the open and closed symbols, respectively. Asterisks show eye movement data obtained when the mask consisted of a 14 cycles/ deg vertical grating, with a space-averaged luminance of $1.0 \mathrm{log} \mathrm{fL}$ (the same average luminance as the uniform mask).

For both real and simulated saccades, threshold sensitivity begins to change to targets presented about 50-75 msec before the onset of movement and the actual step change in luminance. Threshold rises rapidly to a peak that is well above the mask fixation value, begins to fall during the eye movement, and descends gradually toward the background threshold over several hundred milliseconds as the eye steadily regards the background in the postsaccadic period. All the changes are more pronounced for the more intense mask. The peak increase of threshold coincides with the mask offset, and the changes are similar whether produced by a saccade or by simulated saccadic movement of the mask. Furthermore, when the mask consisted of the grating, there was little effect on the detection threshold, although the observer found the grating irritating.

In Figure 3, analogous data are presented for a postsaccadic mask. In this case, the termination of movement results in either a 1.0 - or 3.0 -log-unit luminance increment at the central retina. Dotted lines indicate target threshold values during steady fixation of the mask. There is an increase of threshold to targets presented as much as $170 \mathrm{msec}$ before the mask onset; this is also well before the onset of eye movement. Threshold continues to increase during the eye movement and peaks at the moment of luminance change, reaching values that are considerably higher than during steady regard of the mask. After the peak, the threshold gradually falls to a steady level, requiring more time in the case of the stronger mask. Results are comparable for real saccadic and simulated saccadic conditions when the eye was steady.

A strong temporal asymmetry in backward and forward masking can be seen in the results of Figures 2 and 3. Whether the mask is an incremental or decremental step, thresholds are affected for longer times after the step change (forward effect) than before (backward effect). It is remarkable that an increase in threshold is found regardless of the direction of mask luminance change (i.e., target sensitivity decreases regardless of whether the mask is an increase or decrease of luminance). Such effects on sensitivity have been described in earlier studies of threshold behavior at the onset and offset of an adapting background (Baker, 1963; Crawford, 1947).

\section{The Influence of Target Intensity on Masking Duration}

Figures 2 and 3 illustrate a positive correlation between the amount and duration of masking and the intensity of the mask. It might then be predicted that if mask intensity is constant, weak targets should be masked over a longer time than more intense targets. This experiment was performed with duration of masking as the dependent variable, and the results were according to expectation.

Target intensities were set at $1.1,1.6$, or $2.1 \mathrm{log}$ units above threshold on the $-2.0-\log$-fL background The mask was set at 3.0 log units above background 
Table 1

Threshold Target-Mask Intervals (T-MI) in Milliseconds for a Constant-Intensity Mask $(1.0 \log \mathrm{fL})$ With Different Target Intensities

\begin{tabular}{|c|c|c|c|c|c|}
\hline \multirow{3}{*}{$\begin{array}{c}\text { Target } \\
\text { Intensity }\end{array}$} & \multicolumn{4}{|c|}{ Threshold T-MI } & \multirow[b]{3}{*}{ Observer } \\
\hline & \multicolumn{2}{|c|}{ Forward Mask } & \multicolumn{2}{|c|}{ Backward Mask } & \\
\hline & Mean & Range & Mean & Range & \\
\hline 2.1 & 22 & $13-35$ & 13 & $8-17$ & B.B. \\
\hline 1.6 & $\begin{array}{l}89 \\
75 \\
74\end{array}$ & $\begin{array}{l}84-95 \\
71-86 \\
60-82\end{array}$ & $\begin{array}{l}27 \\
28 \\
31\end{array}$ & $\begin{array}{l}20-37 \\
20-39 \\
24-39\end{array}$ & $\begin{array}{l}\text { B.B. } \\
\text { D.I. } \\
\text { D.I. }\end{array}$ \\
\hline 1.1 & $\begin{array}{l}337 \\
356\end{array}$ & $\begin{array}{l}317-386 \\
330-397\end{array}$ & $\begin{array}{l}49 \\
54\end{array}$ & $\begin{array}{l}45-58 \\
40-63\end{array}$ & $\begin{array}{l}\text { B.B. } \\
\text { B.B. }\end{array}$ \\
\hline
\end{tabular}

Note-Masking was caused by real saccades except for data in italics, which were obtained in simulated saccadic conditions. Range is given in milliseconds from $0 \%$ to $100 \%$ detection.

(at $1.0 \log \mathrm{fL}$ ). Each target intensity was presented either during or after a $15-\mathrm{deg} 45-\mathrm{msec}$ eye movement with the mask in the presaccadic position (for forward effects) or before or during eye movement with the mask in the postsaccadic position (for backward effects). The T-MI was varied in each condition to yield a complete frequency of seeing curve, with threshold (in milliseconds) defined as the T-MI that permitted $50 \%$ detection.

Table 1 shows that in both forward and backward masking conditions, weaker targets (e.g., at 1.1 log units above threshold) were masked over longer intervals than were more intense targets (2.1 log units above threshold). At all target intensities, backward masking extended over shorter durations than forward masking. Depending on target intensity, backward masking from a postsaccadic mask could end during the preceding saccade or extend into the presaccadic period, analogous to Figures 2 and 3. There is good agreement between observers at the target intensity tested for both (1.6 log units above threshold). The contribution of eye movement per se to these data was coincidental, as shown by very similar measures obtained during simulated saccadic controls (data in italics). Once again, none of the measures were affected by introducing a 14-cycles/deg vertical grating into the mask (data not shown).

\section{No Influence of Eye Movement Parameters}

The direction of eye movement was immaterial to any of the effects we measured, as long as the stimulus conditions were held constant. It was also of interest to determine the influence, if any, of eye movement amplitude upon the amount of masking. We kept the mask intensity and T-MI constant while varying the intensity of the target at several different amplitudes (and velocities) of eye movement. The mask was set at $0.0 \log \mathrm{fL}$ in either the presaccadic or postsaccadic position, and the EOG trigger voltage level was ar- ranged for the different eye movement amplitudes to give a constant T-MI (about $43 \mathrm{msec}$ ). Each eye movement and its trigger pulse were recorded on a high-speed CRT chart recorder (Honeywell Visicorder, Model 1858) and T-MIs were analyzed individually.

Figure 4 shows the results of this experiment; the legend includes the mean and standard deviations of T-MIs obtained in each condition. Data in the backward mode are for targets triggered at approximately $43 \mathrm{msec}$ before the fovea landed on a postsaccadic mask. Since the eye movements were of different durations, it was necessary to vary the EOG trigger position from early in the movement (21-deg saccade $=55 \mathrm{msec}$ total duration) to about three-fifths of the way through (41-deg saccade $=95 \mathrm{msec}$ total duration). The trigger level of the EOG was also varied in the forward mode so that the target was presented

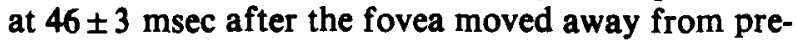
saccadic mask. (The target always stimulated the center of gaze.) It can be seen that, regardless of eye movement amplitude, the target had to be increased approximately $1.25 \log$ units to be visible $50 \%$ of the time in the backward condition. In contrast, the intensity increase necessary for $50 \%$ visibility in the forward condition was about $1.8 \mathrm{log}$ units, indicating a more potent masking effect. None of the results were significantly influenced by eye movement amplitude.

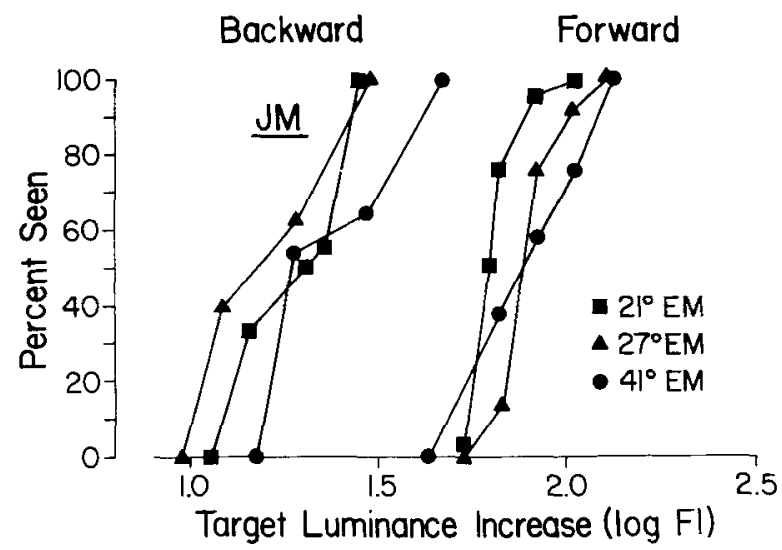

Figure 4. Detection of target at constant target-mank intervals, but different eye movement amplitudes $(21,27,41$ deg). Ordinate: Percent correct tanget detection, with at least 30 trials per data point. Abacisan: Luminance of target relative to target threshold on the uniform background without the mask ( 0 on absciana). Control trial performance was better than $90 \%$ correct in all conditions. Backward and forward masks were the same intensity $(.0 \log \mathrm{n})$. Target-mask intervals were as follows: Backward (postsaccadic)EM $21 \mathrm{deg}$, mean $=40.8 \pm 3.4 \mathrm{msec}$; EM $27 \mathrm{deg}$, mean $=44.5 \pm$ 3.1 msec, EM $41 \mathrm{deg}$, mean $=42.0 \pm 3.8$ msec. Forward (presaccadic)-EM 21 deg, mean = $45.0 \pm 2.5$ msec; EM 27 des, mean = $46.7 \pm 2.7$ msec; EM 41 deg, mean $=46.0 \pm 3.9$ msec. Overlap of curves indicates that detection was dependent on T-MI and not on eye movement amplitude. 


\section{DISCUSSION}

The quantitative similarity between results obtained during real saccades and in simulated saccadic conditions demonstrates that masking associated with eye movement was completely determined by parameters of stimulation of the retina. The presence of eye movement was totally coincidental to the data and served only as a means to produce the retinal stimulus sufficient for masking. Other factors that have been suggested to reduce sensitivity during saccades include mechanical disturbances at the retina (Richards, 1969) and suppressive extraretinal influences (corollary discharge, etc.; see reviews by Matin, 1974; Volkmann, 1976). To the extent that such factors may exist, they are certainly not additive with the influence of visual masking. The fact that few or no differences were found between the moving and steady eye regarding luminance thresholds for the target in the absence of the mask is consistent with the lack of evidence for nonvisual factors in the masking data (see Appendix).

For the stimuli we used, the duration of masking in a forward direction is longer than in a backward direction. ${ }^{2}$ Nevertheless, the time course of backward masking may extend well before eye movement (for discussions of backward masking associated with eye movement, see Brooks \& Fuchs, 1975; Grusser, 1972; MacKay, 1970; Matin, 1974; Matin, Clymer, \& Matin, 1972; and Volkmann, 1976). It is possible that the asymmetry in forward and backward masking pertains only to targets of low spatial frequency; quite possibly targets of higher spatial frequency might show a different time course of sensitivity change when competing against masks of high- or low-frequency composition.

Threshold increases were caused by both increment and decrement in the mask luminance. The threshold begins to rise for stimuli presented $30-200 \mathrm{msec}$ before the luminance step (depending on the direction and intensity of the step), peaks at approximately the moment of luminance change and drops more slowly to a steady state level. These effects require that the mask and target affect the same retinal area, since they could be demonstrated only if the mask overlapped the target (observers could immediately perceive targets that, through some timing or positional error, did not fall on retina affected by the mask). The results are comparable to Crawford's (1947) earlier studies on the time course of light adaptation in the fixating eye. Both Baker $(1953,1963)$ and Crawford (1947) described increases in threshold for light flashes at the onset and offset of an adapting luminance, including a sharp rise just before the luminance change occurred. Holzworth and Doherty $(1971,1974)$ have shown that thresholds for patterned targets are also increased at both "on" and "off" of a lighted background. The significance of our results for ordinary eye movements is that any luminance shift at the retina will momentarily decrease visual sensitivity, at least for targets of our type. Such shifts are caused every time a saccade is made in normal environments. The potential significance for visual processing and a possible neural basis for the interactions we have seen is discussed below.

\section{The Role of $X$ and $Y$ Channels in Target-Mask Interactions}

Our target was a nonpatterned and slightly defocused flash intended to affect primarily the low-resolution channels of the visual system specialized for the detection of low spatial frequencies, movement, and temporal change ("Y cells" or "transient"' channels of the literature, as compared with the "X" cells, stationary pattern detecting, high spatial frequency, or "sustained" channels; see review by Breitmeyer \& Ganz, 1976). At threshold, the target had no "shape," and it was difficult to decide whether it was an increase of decrease in luminance. The masking stimulus (a step change in luminance) was also of the type registered by $Y$ cells of the visual system, many of which respond at both on and off phases to a light stimulus (Breitmeyer \& Ganz, 1976). Since the target and mask affected the same retinal area and probably the same neuronal population, it is conceivable that the neuronal response to the weak (near-threshold) target was lost in or could not compete with the response to the stronger mask. In the case of backward masking, longer latency target responses may have been overtaken at an early point in the visual pathway by short latency mask responses. These represent within-channel interactions. On the other hand, the presence of high-frequency gratings $(14 \mathrm{cycles} / \mathrm{deg})$ in the mask (either pre- or postsaccadic) affected neither the amount nor the time course of target masking: Target detection was at all times, whether during fixation or eye movement, a function only of space-averaged mask luminance. This suggests that activation of movement detecting $(Y)$ channels is not affected by simultaneous of near-simultaneous activity in pattern detecting (X) channels. Supporting this conclusion is the report of Brooks, Impelman, and Lum (1980) that light flash threshold during saccades are unaffected by high-frequency gratings continuously present in the visual background across which the saccades are made. Our data do not permit the conclusion that pattern-detecting channels are unaffected by activity in the " $Y$ " channels; on the contrary, it has been speculated that $X$ channels may register the clear images of fixation and that $Y$ channels may inhibit the $X$ channels during saccadic movement, so as to cut off potential forward masking effects from the presaccadic scene and "clear" the $X$ 
channels for the new postsaccadic images (e.g., see review by Breitmeyer \& Ganz, 1976). These ideas await investigation.

\section{REFERENCES}

BAKER, H. D. The instantaneous threshold and early dark adaptation. Journal of the Optical Society of America, 1953, 43, 798-803.

BAKER, H. D. Initial states of light and dark adaptation. Journal of the Optical Society of America, 1963, 53, 98-103.

Blackweld, H. B. Psychophysical thresholds: Experimental studies of methods of measurements. Bulletin of the Engineering Research Institute of the University of Michigan, 1953, 36, 16.

BREITMEYER, B. G., \& GANz, L. Implications of sustained and transient channels for theories of visual pattern masking, saccadic suppression, and information processing. Psychological Review, 1976, 83, 1-36.

Broors, B. A., \& Fuchs, A. F. Influence of stimulus parameters on visual sensitivity during saccadic eye movements. Vision $R e$ search, 1975, 15, 1389-1398.

Broors, B. A., Impelman, D. M., \& Lum, J. L. Influence of background luminance on visual sensitivity during saccadic eye movements. Experimental Brain Research, 1980, 40, 322-329.

Brooks, B. A., Yates, J. T., \& Coleman, R. C. Perception of images moving at saccadic velocities during saccades and during fixation. Experimental Brain Research, 1980, 40, 71-78.

Campbell, F. W., \& Wurtz, R. H. Saccadic omission: Why we do not see a gray-out during a saccadic eye movement. Vision Research, 1978, 18, 1297-1303.

Corfield, R., Frosdick, J. P., \& Campbell, F. W. Gray-out elimination: The roles of spatial waveform, frequency and phase. Vision Research, 1978, 18, 1305-1312.

Crawford, B. H. Visual adaptation in relation to brief conditioning stimuli. Proceedings of the Royal Society (London), 1947, 134B, 283-300.

Davidson, M. L., Fox, M., \& Dick, A. O. Effect of eye movement on backward masking and perceived location. Perception \& Psychophysics, 1973, 14, 110-116.

Grusser, $O$. Metacontrast and the perception of the visual world. European Journal of Physiology (Pflügers Archiv), 1972, 322 (Suppl.), R98.

Harrison, S., \& Harrison, S. J. A psychophysical method employing a modification of the Muller-Urban weights. Psychological Bulletin, 1951, 48, 249-256.

Holzworth, R. J., \& DoherTy, M. E. Visual masking by light offset: An experiment in reply to Hogben and DiLollo. Journal of Experimental Psychology, 1974, 103, 815-816.

Holzworty, J., \& Doherty, M. E. Visual masking by light of fset. Perceptual Psychophysics, 1971, 10, 327-330.

JudGe, S. L., Wurtz, R. H., \& Richmond, B. J. Vision during saccadic eye movement. I. Visual interactions in the striate cortex. Journal of Neurophysiology, 1980, 43, 1133-1155.

Kahneman, D. Method, findings, and theory in studies of visual masking. Psychological Bulletin, 1968, 70, 404-425.

MACKAY, D. M. Mislocation of test flashes during saccadic image displacements. Nature, 1970, 227, 731-733.

Matin, E. Saccadic suppression: $A$ review and an analysis. Psychological Bulletin, 1974, 81, 899-917.

Matin, E., Clymer, A. B., \& Matin, L. Metacontrast and saccadic suppression. Science, 1972, 178, 179-182.

Mitrani, L., Mateeff, St., \& Yakimorf, N. Is saccadic suppression really saccadic? Vision Research, 1971, 11, 1157-1161.

Pearce, D. G., \& Porter, E. Changes in visual sensitivity associated with voluntary saccades. Psychonomic Science, 1970, 19, 225-227.

RAAB, D. H. Backward masking. Psychological Bulletin, 1963, 60, 118-129.
Richards, W. Saccadic suppression. Journal of the Optical Society of America, 1969, 59, 617-623.

Riggs, L. A., Merton, P. A., \& Morton, H. B. Suppression of visual phosphenes during saccadic eye movements. Vision Research, 1974, 11, 997-1011.

TURveY, M. T. On peripheral and central processes in vision: Inferences from an information processing analysis of masking with patterned stimuli. Psychological Review, 1973, 80, 1-52.

Volkmann, F. C. Saccadic suppression: A brief review. In R. A. Monty \& J. W. Senders (Eds.), Eye movements and psychological processes. Hillsdale, N.J: Erlbaum, 1976.

Volkmann, F. C., Riggs, L. A., Moore, R. K., \& White, K. D. Central and peripheral determinants of saccadic suppression. In J. W. Senders, D. F. Fisher, \& R. A. Monty (Eds.), Eye movement and the higher psychological functions. Hillsdale, N.J: Erlbaum, 1978.

Weisstein, N. Metacontrast. In D. Jameson \& L. M. Hurvich (Eds.), Handbook of sensory physiology (Vol. 7, Pt. 4) Visual psychophysics. New York: Springer-Verlag, 1972.

Wolf, W., Hauske, G., \& LUPP, U. How presaccadic gratings modify postsaccadic modulation transfer function. Vision $R e$ search, 1978, 18, 1173-1179.

ZAR, J. H. Biostatistical analysis. Englewood Cliffs, N.J: PrenticeHall, 1974.

\section{NOTES}

1. The psychophysical literature for the fixating eye, using spatially overlapping stimuli characterized by luminance and low spatial frequency (e.g., light flashes), indicates that masking interactions are governed chiefly by the parameters of intensity and temporal interval (for reviews see Kahneman, 1968; Raab, 1963; Turvey, 1973; Weisstein, 1972). Within time limits, the visibility of a briefly flashed luminous target is reduced whether it precedes or follows a mask in time: "backward" masking (target precedes the mask) may extend over shorter intervals than "forward" masking (target follows the mask). The strongest masking occurs when the target and mask occur simultaneously ("Type A" masking of Kahneman, 1968), and mask potency is determined directly by its intensity and inversely by the target intensity. For conditions in which the mask overlaps and is substantially larger than the target, the mask is never itself masked by the target.

2. Following the completion of this study, Judge, Wurtz, and Richmond (1980) published an investigation of pre- and postsaccadic masking in neurons of the monkey striate cortex. Both their neurophysiological results and their psychophysical observations confirm longer durations for forward masking than backward masking.

\section{APPENDIX}

Although several authors (see Results) have reported no significant difference in visual sensitivity during eye movements when compared with fixation at dim ambient luminance, Riggs et al. (1974) described a slight threshold elevation for phosphenes during rapid eye movements in the dark, which they estimated would be equivalent to an approximate .4-log-unit change in threshold luminance. This apparent paradox might be due to the fact that Riggs et al. (1974) alone used the double-alternation forced-choice technique preferred by signal detection theorists; the other studies did not. To resolve this question, we compared thresholds during eye movement and when the eyes were steady, using a uniform background of $-2.0 \log \mathrm{fL}$, and the stimulation methods previously described. Two psychophysical procedures were compared: a yes-no $(\mathrm{Y} / \mathrm{N})$ pro- 
cedure similar to that described in the Procedure section and a double-alternation forced-choice routine (2AFC) favored by signal detection theorists.

In $\mathrm{Y} / \mathrm{N}$ procedures, observers were required to report whether or not they had detected the flash immediately following each trial. A total of 40-45 trials for Observer B.B. (age 45) and 50-80 trials for Observer J.L. (age 23, graduate student) constituted an experimental run at a single flash intensity randomly chosen by the experimenter and unknown to the subject (since J.L. was the less experienced subject, a greater number of experimental trials were used to assess her sensitivity). Equal numbers of flash and no flash-control trials were interspersed randomly in each experimental run. During preliminary practice and also during experimental runs, the experimenter corrected the observer each time she reported a "yes" answer on a no-flash trial. With this procedure, observers were able to maintain a false alarm rate of less than $5 \%$ and therefore to minimize the effect of criterion shifts on the $\mathrm{Y} / \mathrm{N}$ data (Pearce \& Porter, 1970).* The observers' performance was scored by calculating the percentage of flashes eliciting a "yes" response out of the total number of flashes presented at a given intensity. These data were plotted as frequency of seeing curves (percent correct as a function of flash intensity), and the intensity seen $50 \%$ of the time was defined as threshold. A percent correct score was also obtained on no-flash control trials, in this case being the proportion of "no" answers in the total number of control trials. Ninetyfive percent confidence intervals were calculated for each data point, and regression lines were fitted to the various curves.

In the 2AFC method, paired trials were given, only one of which contained the target flash. After each trial pair, the observer was required to report on which trial the flash occurred, even if she had not detected the flash. A random sequence of flash presentations for a series of 100 paired trials at each intensity was generated from a random number computer program. The percent observed $\left(P_{0}\right)$ was scored as the percentage of flashes detected out of the total number of flashes presented. The percent correct $\left(P_{c}\right)$ was obtained by the formula $P_{c}=2\left(P_{0}-.50\right)$, which adjusts for the percentage of correct observed responses that may occur by chance (Blackwell, 1953; Harrison \& Harrison, 1951). Threshold was defined as the flash intensity detected on $50 \%\left(P_{c}\right)$ of the trials. Ninety-five percent confidence intervals and regression lines were also calculated for these data.

Figures $\mathrm{A} 1$ and $\mathrm{A} 2$ compare threshold estimates obtained by the $Y / N$ and 2AFC methods in conditions of eye movement and while the eyes were held steady ("fixation" condition). Data for B.B. (Figure A1) were obtained when the test flash occurred 10 deg above the fovea (peripheral retina); for J.L. the flash was positioned to affect the center of gaze.

\footnotetext{
* These authors showed that when $\mathrm{Y} / \mathrm{N}$ data were corrected to a $0 \%$ guess rate, they were comparable to the bias-free $2 \mathrm{AFC}$ method. 2AFC data for Observer B.B. were not significantly different from her $\mathrm{Y} / \mathrm{N}$ data obtained with an actual $0 \%$ guess rate (see Figure A1). J.L.'s data were corrected by the method of Pearce and Porter, but the corrected values did not significantly alter the main findings summarized for her original data, which showed some differences between results obtained by $2 \mathrm{AFC}$ and Y/N methods.
}

PERIPHERAL RETINA (BB)
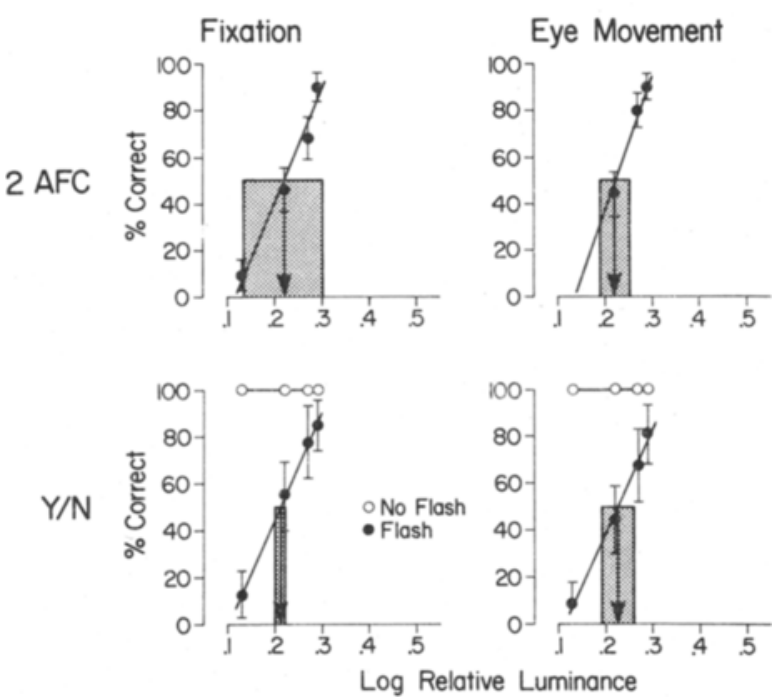

Figure A1.

\section{CENTRAL RETINA (JL)}

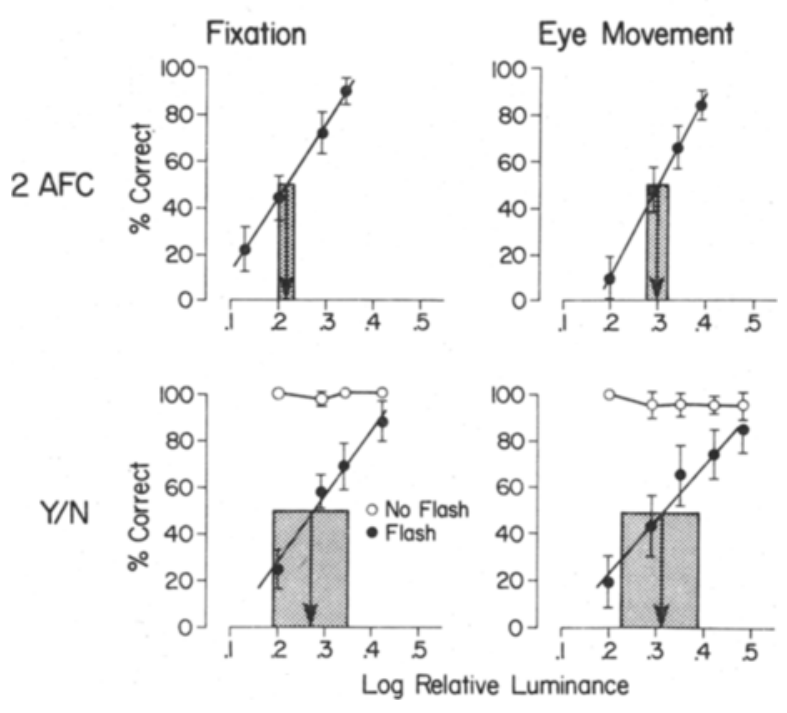

Figure A2.

For both observers, the flash occurred $20-25 \mathrm{msec}$ after saccade onset. The ordinates represent percent correct as discussed earlier; the abscissas denote a common log luminance scale based on the range of neutral density filters used in the experiments and expressed in arbitrary units. Arrows indicate interpolated threshold values with their $95 \%$ confidence intervals in dark stipple.

The slopes and intercepts of calculated regression lines were compared to determine differences between experimental conditions. This treatment was justified on the grounds that the mid-range of "sigmoidal" frequency of 
Table A1

\begin{tabular}{lcc}
\hline Conditions Compared & t (slopes) & $t$ (intercepts) \\
\hline Y/N Fix / Y/N EM & 1.38 & 1.10 \\
Y/N Fix / 2AFC Fix & 1.28 & $13.30^{*}$ \\
Y/N EM / 2AFC EM & $4.45^{*}$ & $10.84^{*}$ \\
2AFC Fix / 2AFC EM & $5.13^{*}$ & $63.94^{*}$ \\
2AFC Fix / Y/N EM & 2.78 & $10.60^{*}$ \\
2AFC EM/ Y/N Fix & 3.37 & $15.27^{*}$ \\
\hline
\end{tabular}

*Significant at the .01 level for a two-tailed t test.

seeing data is linear or nearly linear, and our tests of linearity showed a good fit for the regression lines (the pooled standard error of the regressions was $\pm 3.3 \%$ ). Statistical analysis for coincidence of regression lines (Zar, 1976) showed that, for the more experienced observer (B.B.), there was no significant difference in visual sensitivity and therefore in the threshold for seeing under the two conditions as measured by either method. This was not the case for the less experienced observer (J.L.), for whom the 2AFC method showed a small (.08 log unit) but significant difference between the fixation and eye movement thresholds. The $\mathrm{Y} / \mathrm{N}$ method did not show any significant difference for J.L.; furthermore, visual sensitivity under each condition as measured by the two methods was also significantly different. Statistical differences between the six possible pairs of regression lines obtained for J.L.'s data are shown in Table A1, which lists " $t$ " values calculated for their slopes and intercepts.

The main point here is that the 2AFC method does show a significant difference between eye movement and fixation thresholds, but the $\mathrm{Y} / \mathrm{N}$ method does not. There is also less variability in the threshold values interpolated from the 2AFC curves than for those obtained from $\mathrm{Y} / \mathrm{N}$ data for the less experienced observer. Even so, threshold values obtained for J.L. do not vary more than .1 log unit relative to the .2-.3-log-unit change required to increase detection rate from $10 \%$ to $90 \%$ for this observer. Therefore, these differences would seem to be of questionable biological significance. In this same context, it is equally difficult to see how an .08-log-unit threshold elevation during eye movements could be in agreement with a .4-log-unit increase estimated by Riggs et al. (1974) during saccades made in the dark. J.L.'s data are in closer agreement with those reported by Brooks and Fuchs (1975) and Richards (1979) who sometimes, but not always, found small (.1-log-unit) and not always significant elevation in saccadic threshold at dim backgrounds. Differences between our observers may be attributable to their relative facility in making threshold judgments during eye movements. The well-trained observer made regular eye movements with less effort than did the less experienced observer and therefore was able to attend to the stimulus situation more closely. This interpretation would be consistent with the fact that Observer J.L. preferred the $2 \mathrm{AFC}$ method, which did not require that she perform to $90 \%$ criterion on no/flash trials as in the $Y / N$ method (B.B. preferred the $\mathrm{Y} / \mathrm{N}$ method, which took only half the time required for the 2AFC).

From these data, we conclude that, while there is some evidence for systematic differences between the two psychophysical methods employed with less experienced observers (see discussion by Blackwell, 1953), there are no convincing differences between sensitivity measured during saccades and sensitivity measured when eyes are steady at dim backgrounds.

(Manuscript received June 24, 1980; revision accepted for publication March 15, 1981.) 